\title{
Gated Graphene Electrical Transport Characterization
}

\author{
Josef Náhlík ${ }^{1}$, Michal Janoušek¹, Zbyněk Šobáň ${ }^{1,2}$ \\ ${ }^{1}$ Dept. of Microelectronics, Faculty of Electrical Engineering, Czech Technical University in Prague, Technická 2, 166 27 \\ Praha, Czech Republic \\ ${ }^{2}$ Institute of Physics ASCR, Cukrovarnická 10/112, 162 00 Praha, Czech Republic
}

Corresponding author: nahlijo1@fel.cvut.cz

\begin{abstract}
Graphene is a very interesting new material, and promises attractive applications in future nanodevices. It is a $2 \mathrm{D}$ carbon structure with very interesting physical behavior. Graphene is an almost transparent material that has higher carrier mobility than any other material at room temperature. Graphene can therefore be used in applications such as ultrahigh-speed transistors and transparent electrodes. In this paper, we present our preliminary experiments on the transport behavior of graphene at room temperature. We measured the resistivity of Hall-bar samples depending on gate voltage (backgated graphene). Hysteresis between the forward and backward sweep direction was observed.
\end{abstract}

Keywords: Graphene, Hysteresis in electric transport.

\section{Graphene}

Graphene is a very interesting new material that was first prepared by A. K. Geim and K. S. Novoselov in 2004 [9]. Graphene is a monolayer of a carbon atom in a honeycomb lattice, and can be prepared in various ways. Graphene has very interesting physical behavior, which promises applications in nanodevices such as ultra-high speed transistors and transparent electrodes.

\subsection{Methods of preparation}

The first preparation method is exfoliation, which was published by Geim and Novoselov. It is a very simple technique for preparing graphene "flakes" from highly-oriented pyrolytic graphite (HOPG), but the dimensions of the "flakes" are very small - about tens $\mu \mathrm{m}$.

The second method is growth by Chemical Vapor Deposition (CVD) on a copper or nickel foil [5, 7, 8]. This method is based on thermal decomposition of methane at high temperature.

The third method is high temperature annealing of silicon carbide in an argon atmosphere or in another inert gas or in a vacuum [3]. It is better to use a semi-isolating silicon carbide for easy electrical characterization. It is important to use $\mathrm{SiC}$ in a specific orientation.

\subsection{Basic properties of graphene}

Graphene is an interesting material not only for its electrical properties, but also for its mechanical and optical properties.
In this paper we will focus on its electrical properties. Graphene is composed of an $\mathrm{sp}^{2}$ bonded carbon atom with lattice constant $\mathrm{a}_{c-c}=1,42 \AA$ (carbon to carbon length). The remaining $\mathrm{p}$ orbitals create $\pi$ bonds responsible for the dominant planar conduction phenomena.

The energy-momentum dispersion relation in the vicinity of the $\mathrm{K}$ point of the Brillouin zone is linear, and the conduction and valence band overlap at one point (Dirac point). Due to this specific relation, charge carriers are seen as zero mass relativistic particles with effective "speed of light", $\mathrm{c} \sim 10^{6} \mathrm{~m} / \mathrm{s}$ [11].

The type of conductivity is related to the dispersion relation. As shown in Fig. 1, the change in Fermi energy when the gate voltage is applied changes the type of conductivity. When the Fermi level matches the Dirac point, the conductivity has a minimum value due to lack of free carriers.

The carrier mobility is also very high. It can be more than $10^{6} \mathrm{~cm}^{2} / \mathrm{Vs}$, but the charge mobility depends on many parameters. The main influences include the concentration of impurities, the roughness of the substrate, and temperature. The highest measured charge mobility is about $2 \times 10^{5} \mathrm{~cm}^{2} / \mathrm{Vs}$ [1] This is more than one hundred times higher than in silicon.

\subsection{Identification methods}

Graphene is a very thin material and its optical transparency is very high $[10,11]$ (monolayer - nearly $98 \%$ for visible wavelengths). It is therefore almost invisible on a transparent material. Interference with 


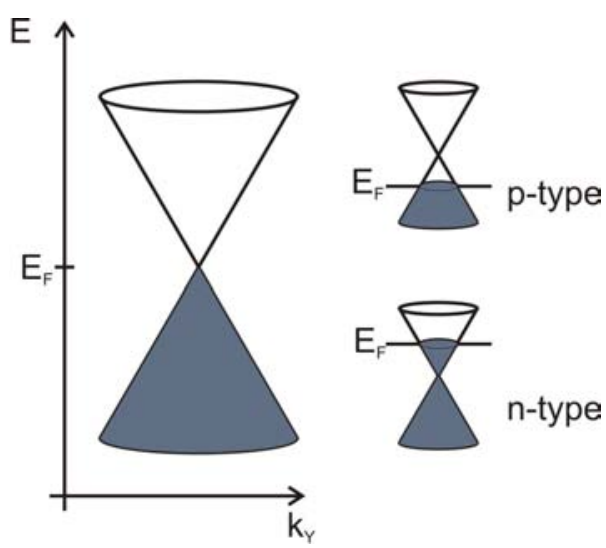

Figure 1: Ambipolar electric field effect in singlelayer graphene. The positive (negative) gate voltage changes the Fermi energy and the induced electrons (holes), and causes electron (hole) conductivity.

the substrate is used for optical identification. A silicon substrate with silicon dioxide $90 \mathrm{~nm}$ or $300 \mathrm{~nm}$ in thickness, where the optical contrast between the monolayer of graphene and the substrate can be as high as $12 \%$, is preferred for this reason.

The most reliable method for confirming that that examined material is graphene is Raman spectroscopy, which is a noninvasive technique for identifying the composition of the studied material. A carbon monolayer has its own unique spectrum (multilayer graphene or graphite has a different Raman spectrum) $[2,11]$. Our graphene samples were checked by this technique.

Indentification can be performed by AFM (Atomic Force Microscopy). The height of the graphene layer on an oxidized silicon substrate is not $0,35 \mathrm{~nm}$, i.e. the interlayer distance of graphite. The height is $0,8^{-}$ $1,2 \mathrm{~nm}$, due to the native van der Waals inter-layer distance [11].

\section{Electrical hysteresis in graphene}

The gate voltage that is applied changes the Fermi energy position, and consequently also the resistivity of the graphene. Hysteresis between points of maximum resistivity in two different direction sweeps of the gate voltage has been observed. This phenomenon is most often explained by the influence of the ambient air humidity $[4,6,12]$.

A layer of silanol groups $(\mathrm{SiOH})$ is formed on the surface of the silicon dioxide substrate $\left(\mathrm{SiO}_{2}\right.$ on silicon), and attracts $\mathrm{OH}$ groups (or other molecules). Dipolar water molecules are easily absorbed, and they can influence the charge transfer. Water molecules behave as doping atoms, because the gate voltage affects their polarization and they add their own electric field intensity to the intensity caused by the

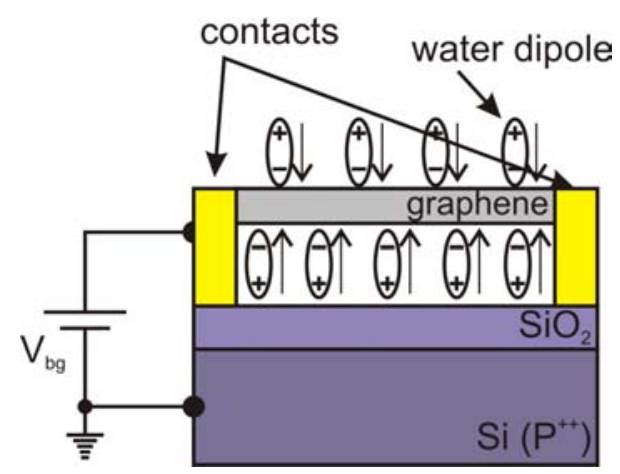

Figure 2: Scheme of the polarization of water molecules by gate voltage in backgated graphene.

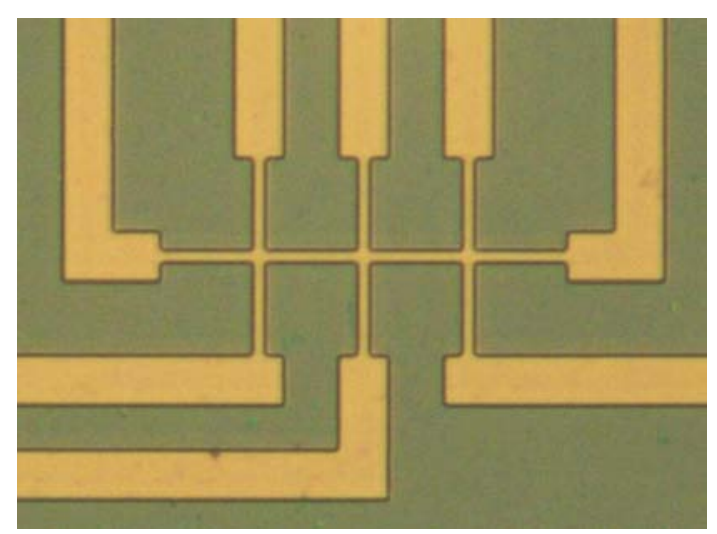

Figure 3: A graphene Hall-bar structure defined by electron lithography.

voltage source that is connected between the conductive substrate and the graphene (so-called backgated graphene). Fig. 2 presents the scheme of polarized water molecules.

Polarized molecules and their electric intensity could be one of the effects that cause hysteresis between the forward and backward sweep of gate voltage in the resistivity/gate voltage characteristic.

Another way to explain hysteresis is by the presence of charge traps from the surface of silanol or surfacebound $\mathrm{H}_{2} \mathrm{O}$ molecules $[4,6]$.

To suppress the influence of water, the substrate can be covered with a hydrophobic hexamethyldisilazane (HMDS) layer [4, 6].

\section{$3 \quad$ Experimental setup}

Our graphene samples were prepared by chemical vapor deposition on a copper foil and then transferred to a silicon substrate with $300 \mathrm{~nm}$ silicon dioxide. The presence of a graphene monolayer was confirmed by Raman spectroscopy. After deposition of the samples, electron lithography was used to define the $2 \mu \mathrm{m}$ wide Hall-bar shown in Fig. 3.

The graphene was etched by oxygen plasma, and the metal contacts $(\mathrm{Cr}-5 \mathrm{~nm}, \mathrm{Au}-100 \mathrm{~nm})$ were fab- 


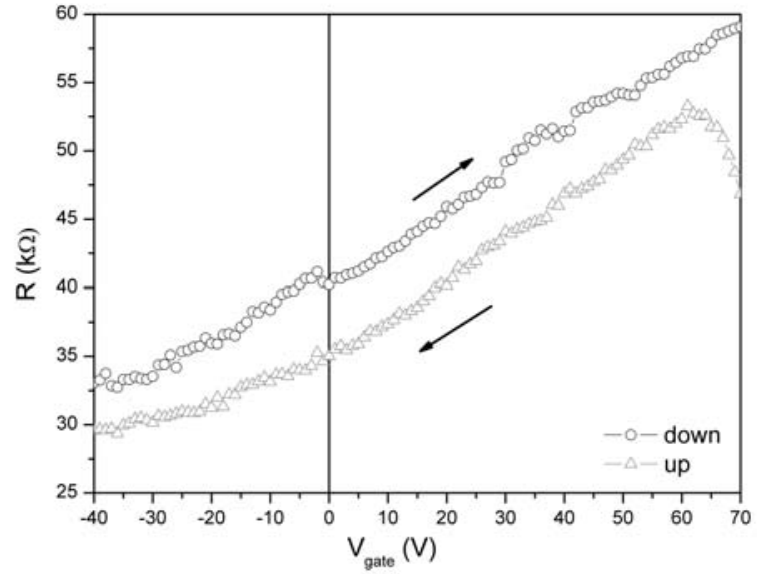

Figure 4: Hysteresis behavior for low-speed gating (15-second applied gate voltage before measuring VA characteristics).

ricated by standard UV lithography. Thereafter the samples were placed into ceramic chip carriers and were wirebonded.

The graphene samples were electrically characterized using an HP4156C Semiconductor Parameter Analyzer. Resistivity measurements of the graphene Hall-bar vs. gate voltage were performed in a fourpoint configuration at room temperature. The samples were measured using a constant current source. The gate voltage was connected to the doped silicon substrate.

For each gate voltage we measured about 10 points of VA characteristics to confirm that each of the characteristics is linear.

\section{Results and discussion}

The gate voltage was varied in the $-40 \mathrm{~V}$ to $70 \mathrm{~V}$ range. The dependence of resistivity on the gate voltage of the graphene samples is shown in Fig. 4. The characteristics exhibit hysteresis between the forward and backward sweep directions, as we had assumed. As shown in Fig. 4, only one Dirac point was reached.

The dependence on sweep rate was observed on this sample [4, 12]. The low speed gating (15-second applied gate voltage before measuring the VA characteristics) exhibits a smaller difference between the resistivity of the forward and backward sweep direction than the higher speed gating (5-second applied gate voltage), which is shown in Fig. 5.

\section{Conclusion}

We have presented a preliminary study of graphene transport behavior. We prepared samples that were checked by Raman spectroscopy.

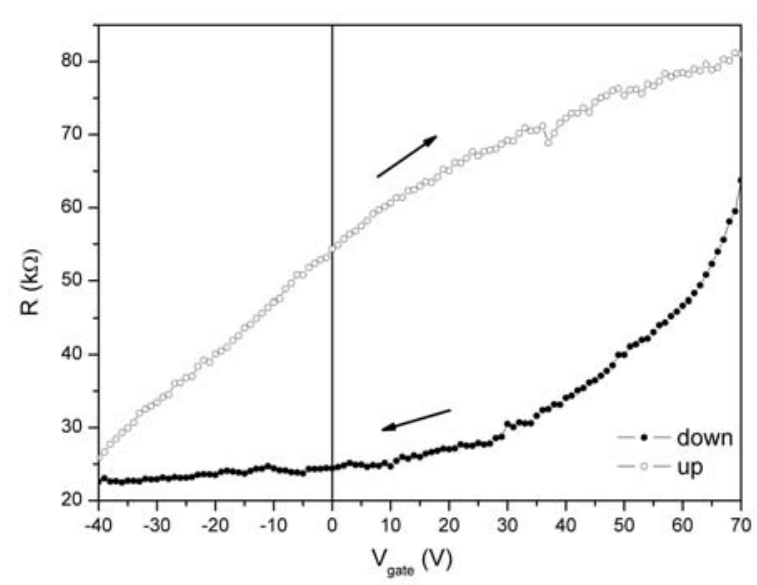

Figure 5: Hysteresis behavior for higher-speed gating (5-second applied gate voltage before measuring VA characteristics).

The dependence between resistivity and gate voltage was measured, and hysteresis between forward and backward sweep directions was shown. The influence of the sweep rate was also investigated.

For our future work, a new set of samples will be covered with a hydrophobic HMDS layer to reduce the influence of air humidity. A different way will be used for depositing the HMDS layer.

\section{Acknowledgements}

The research presented in this paper was supervised by Assoc. Prof. J. Voves, FEE CTU in Prague, and was supported by GACR grant No. P108/11/0894 and by the Grant Agency of the Czech Technical University in Prague, grant No. SGS10/281/OHK3/3T/13.

\section{References}

[1] Bolotin, K.I., et al. Ultrahigh electron mobility in suspended graphene. Solid State Communications 146:351-355, 2008.

[2] Calizo, I., et al. Temperature dependence of raman spectra of graphene multilayers. Nano Lett 7 (9):2645-2649, 2007.

[3] Emstev, K. V., et al. Towards wafer-size graphene layers by atmospheric pressure graphitization of silicon carbide. Nature Materials 8:203, 2009.

[4] Joshi, P., et al. Intrinsic doping and gate hysteresis in graphene field effect devices fabricated on $\mathrm{sio}_{2}$ substrates. J Phys: Condens Matter 22:334214, 2010.

[5] Kim, K. S., et al. Large-scale pattern growth of graphene films for stretchable transparent electrodes. Nature 457:706, 2009. 
[6] Lafkoti, M., et al. Graphene on a hydrophobic substrate: Doping reduction and hysteresis suppression under ambient conditions. Nano Lett 10 (4):1149-1153, 2010.

[7] Li, X., et al. Large-area synthesis of high-quality and uniform graphene films on copper foils. Science 324:1312, 2009.

[8] Mattevi, C., Kim, H., Chhowalla, M. A review of chemical vapour deposition of graphene on copper. J Mater Chem 21:3324, 2011.

[9] Novoselov, K.S., et al. Electric field effect in atomically thin carbon films. Science 306:666, 2004.
[10] Orlita, M., Potemski, M. Dirac electronic states in graphene systems: optical spectroscopy studies. Semicond Sci Technol 25:063001, 2010.

[11] Soldano, C., Mahmood, E., Dujardin, E. Production, properties and potential of graphene. Carbon 48:2127, 2010.

[12] Wang, H., et al. Hysteresis of electronic transport in graphene transistors. ACS Nano 4 (12):72217228, 2010. 